\title{
A investigação em comunicação organizacional no Brasil: uma leitura abrangente dos grupos de pesquisa cadastrados no $\mathrm{CNPq}$ Investigation in organizational communication in Brazil: a comprehensive approach on research groups registered in CNPq
}

\section{Wilson Costa Bueno}

Doutor e Mestre em Ciências da Comunicação pela Escola de Comunicações e Artes de São Paulo da Universidade de São Paulo (ECA/USP); Professor do Programa de Pós-Graduação em Comunicação Social da Universidade Metodista de São Paulo (UMESP) São Bernardo do Campo (SP), Brasil. E-mail: wilson@comtexto.com.br

\section{Resumo}

Mapeamento e análise dos grupos de pesquisa em comunicação organizacional no Brasil, cadastrados no Diretório dos Grupos de Pesquisa do Conselho Nacional de Desenvolvimento Científico e Tecnológico (CNPq). Além da descrição dos grupos (localização, data de constituição, campo de formação dos seus integrantes, vínculos institucionais, produção etc.), o estudo destaca a importância do líderes, a fragmentação do esforço de pesquisa, a presença majoritária das pesquisadoras, a relação com as universidades públicas e a supremacia das regiões sul e sudeste do Brasil.

Palavras-chave: comunicação organizacional; pesquisa em comunicação organizacional; diretório dos grupos de pesquisa do CNPq; grupos de pesquisa em comunicação organizacional.

\begin{abstract}
Mapping and analysis of the research groups in organizational communication in Brazil, registered in the Directory of Research Groups of the National Council for Scientific and Technological Development (CNPq). This study includes the description of the groups (location, date of incorporation, field training of its members, institutional linkages, production, etc.) and highlights the importance of leaders, the fragmentation of research effort, the majority presence of female researchers, the relationship with public universities and the supremacy of the South and Southeast regions.
\end{abstract}

Keywords: organizational communication; research in organizational communication; directory of research groups of CNPq; research groups in organizational communication. 


\section{A investigação em comunicação organizacional no Brasil: uma leitura abrangente dos grupos de pesquisa cadastrados no $\mathrm{CNPq}$}

\section{Introdução}

A pesquisa em Comunicação no Brasil tem sido contemplada, ao longo do tempo, com um conjunto significativo de obras, especialmente nas duas últimas décadas, quando o campo efetivamente se consolidou com a implantação de dezenas de Programas de Pós-Graduação em Comunicação Social. Em boa parte dos casos, estas obras estiveram focadas em aspectos gerais, prioritariamente metodológicos, buscando traçar um panorama histórico da Comunicação sob o ponto de vista dos esforços de investigação, com o detalhamento de métodos e técnicas particulares (análise de discurso, análise de conteúdo, entrevistas, sondagens de opinião, grupos focais, elaboração de questionários, pesquisa participante e mesmo da estatística aplicada às Ciências Sociais). Estes trabalhos estiveram sob a responsabilidade quase sempre de autores originalmente de outras áreas ou de estrangeiros.

A pesquisa essencialmente qualitativa, com grande prestígio nas áreas de Ciências Humanas e Sociais (e também em Comunicação), pode ser acompanhada em textos de referência importantes como os de Lefèvre (2003), Silverman (2009), Hérbet (2005), Rey (2005) e Poupart (2008).

Esses trabalhos têm sido utilizados recorrentemente para subsidiar a pesquisa em Comunicação em subáreas ou competências determinadas, como Relações Públicas, Jornalismo, Propaganda/Publicidade etc., mas necessariamente não são generosos em exemplos ou análises que se reportam especificamente à prática da comunicação organizacional. Mais recentemente, sobretudo neste século, começaram a ser publicados trabaIhos que se endereçam para aspectos específicos deste campo, como a avaliação do retorno dos investimentos em Comunicação, os métodos de pesquisa na internet e mesmo as métricas utilizadas para mensurar a presença e a imagem das organizações (e das marcas) nas redes e mídias sociais.

Dentro deste breve cenário sobre a pesquisa em Comunicação, com atenção direta ou indireta sobre a pesquisa em comunicação organizacional, algumas obras merecem ser mencionadas. São exemplos deste esforço os trabalhos de Maria Immacolata Lopes (1990), de Ada Dencker e Sarah da Viá (2001), de Lucia Santaella (2001) e o organizado por Jorge Duarte e Antonio Barros (2205).

A obra Pesquisa em Comunicação, de Maria Immacolata Vassalo Lopes, cuja primeira edição circulou em 1990 é, certamente, um dos textos com esta preocupação abrangente, pioneira em nosso país. O texto relaciona alguns paradigmas teórico-metodológicos e a pesquisa em Comunicação Social, destaca o papel da pesquisa na universidade, particularmente nos programas de pós-graduação, e debate as problemáticas metodológicas na prática da pesquisa em Comunicação. Naquele momento, apenas cinco Programas de Pós-Graduação em Comunicação estavam em atividade no Brasil e a pesquisa estava basicamente vinculada aos projetos dos docentes e alunos tendo em vista a sua titulação.

No inicio deste século, merece registro o trabalho de Ada de Freitas Manetti Dencker e Sarah Chucid da Viá (2001) - Pesquisa empírica em ciências humanas (com ênfase em comunicação), que aborda a pesquisa empírica em Ciências Humanas, com atenção especial à Comunicação, e tem como objetivo, conforme explícito na sua apresentação, constituir-se em "um verdadeiro curso de pesquisa para ciências humanas e sociais, com aplicação em áreas da Comunicação" (DENCKER e VıÁ, 2001, p. 16). A elaboração do projeto de pesquisa, as principais técnicas qualitativas e quantitativas são o foco central deste texto.

Data de 2001 a obra de Lucia Santaella, Comunicação \& Pesquisa, que apresenta um histórico das teorias, modelos e âmbitos da pesquisa na Comunicação, descreve as etapas de um projeto de pesquisa, além de analisar a importância da pesquisa e do método científico e promover um mapeamento da área da Comunicação.

O trabalho organizado por Jorge Duarte e Antonio Barros, "Métodos e técnicas de pesquisa em Comunicação", com a primeira edição em 2005, reúne dezenas de estudiosos e, embora se refira à pesquisa em Comunicação de maneira geral, tem espaço importante para métodos e técnicas em comunicação organizacional, merecendo menção a contribuição de Margarida Kunsch (Auditoria de comunicação organizacional), de João Curvello (O desvendar das culturas organizacionais) e Wilson Bueno (Auditoria de imagem na mídia).

Não pode ser esquecido outro conjunto de obras que analisa a pesquisa em Comunicação sob uma perspectiva prioritariamente epistemológica, como A pesquisa empírica em comunicação, organizada por José Luiz Braga, Maria Immacolata Vassalo de Lopes e Luiz Claúdio Martino (2010); a de Francisco Rüdiger, Ciência Social crítica e pesquisa em Comunicação (2002) e a organizada por Maria Helena Weber, Ione Bentz e Antonio Hohlfeldt (2002), com a primeira e a última inseridas na coleção de livros da Compós. 


\section{A investigação em comunicação organizacional no Brasil: uma leitura abrangente dos grupos de pesquisa cadastrados no $\mathrm{CNPq}$}

As obras de Galerani (2006) e Yanaze (2010) dizem respeito específicamente ao campo da comunicação organizacional (em suas modalidades institucional e mercadológica) e a elas podem ser acrescentadas as de Phillips (2008), Rosenwlad (2005) e Powell (2011). As redes e mídias sociais também têm sido analisadas sob o ponto de vista do impacto na vida e nos negócios das organizações, e alguns trabalhos apontam para aspectos (metodológicos ou epistemológicos) da investigação nesses novos ambientes como os de Sterne (2011), Terra (2011) e Fragoso (2011).

A pesquisa em comunicação organizacional ganhou grande impulso com a criação da Abrapcorp (Associação Brasileira dos Pesquisadores em Comunicação Organizacional e Relações Públicas) em 2006, "com o objetivo geral de estimular o fomento, a realização e a divulgação de estudos avançados dessas áreas no campo das Ciências da Comunicação." A dinâmica de seus grupos temáticos e a realização do seu congresso anual permitiram, em pouco mais de cinco anos, o aumento da massa crítica e o compartilhamento de estudos e pesquisas na área, com incentivo inclusive à formação de novos pesquisadores. Dezenas de trabalhos com o resultado de pesquisas sobre a temática da comunicação organizacional têm sido apresentados e estão disponíveis para consulta no portal da Associação.

Merecem ainda menção o Grupo de Pesquisa Relações Públicas e Comunicação Organizacional da Intercom (Sociedade Brasileira de Estudos Interdisciplinares da Comunicação), tradicional na área da Comunicação, que há um bom tempo abriga trabalhos nestas áreas, apresentados durante as reuniões da entidade, realizadas em seu congresso anual, sempre no mês de setembro, ou nos simpósios e colóquios também por ela promovidos.

Desde 2010 a Compós (Associação Nacional dos Programas de Pós-Graduação em Comunicação) também dispõe de um grupo de trabalho - Comunicação em contextos organizacionais - e nos seus encontros anuais abre espaço de prestígio para a divulgação de estudos e pesquisas nesta área.

\subsection{A institucionalização dos grupos de pesquisa}

Embora a pesquisa em comunicação organizacional esteja sendo desenvolvida na universidade brasileira há quatro décadas, particularmente pela ação pioneira dos Programas de Pós-Graduação em Comunicação, a institucionalização dos grupos de pesquisa é um fenômeno recente.
Em geral, até há pouco tempo, os projetos de pesquisa em comunicação organizacional estiveram vinculados aos pesquisadores individualmente, que os desenvolviam nas universidades, seja para obtenção de sua titulação acadêmica (mestrado ou doutorado), seja para completar sua carga horária total de docência, sobretudo para os professores em regime de tempo integral.

Quando inseridos em Programas de Pós-Graduação em Comunicação, muitos pesquisadores irradiam os seus conhecimentos e focos de interesse a partir de seus orientandos e tendem, também, como é possível perceber, pelo Diretório de Grupos de Pesquisa do Conselho Nacional de Desenvolvimento Científico e Tecnológico (CNPq), a incluir seus atuais e ex-orientandos em seus grupos, o que é absolutamente compreensível. Há que se considerar, no entanto, que muitos orientandos, após finalizarem sua presença nos Programas de Pós-Graduação em Comunicação, acabam retornando aos seus locais de origem (muitos vêm inclusive de organizações onde exercem funções de gestores em Comunicação) e nem sempre mantêm seu vínculo com a área acadêmica e mesmo com os seus ex-orientadores. Com isso, muitos grupos de pesquisa incorporam frequentemente pesquisadores que têm tendência à redução da sua produção ao longo do tempo, e é possível perceber que em boa parte dos casos são exatamente os pesquisadores, na maioria jovens ou recém titulados, que perderam vínculo institucional com as universidades que os abrigaram durante o período da pós-graduação.

A constituição recente dos grupos de pesquisa, como indica o Diretório do CNPq, justifica a reduzida produção bibliográfica (livros, capítulos de livros, artigos em revistas acadêmicas ou científicas etc.) em determinados grupos. Entende-se portanto que sua visibilidade é maior apenas por ocasião dos eventos da área, promovidos por entidades que incentivam a divulgação dos resultados de pesquisa (Abrapcorp, Compós, Intercom e até congressos de produção científica realizados internamente nas universidades).

Podemos, no entanto, destacar o pioneirismo da pesquisa da professora Cláudia Peixoto de Moura (2011), apresentado no I Congresso Mundial de Comunicação Iberoamericana, promovido pela Confibercom (Confederación Iberoamericana de Asociaciones Científicas y Acadêmicas de la Comunicacion) em 2011. Neste trabalho a autora "apresenta uma proposta metodológica para análise das práticas acadêmicas dos grupos de pesquisa brasileiros, registrados no CNPq", que também incluiu levantamento e análise, como fizemos 


\section{A investigação em comunicação organizacional no Brasil: uma leitura abrangente dos grupos de pesquisa cadastrados no $\mathrm{CNPq}$}

neste estudo, a partir das informações existentes nesta base de dados.

Cláudia Moura, no mapeamento realizado entre 2009 e 2011, encontrou 40 grupos de pesquisa ${ }^{1}$ e deu particular atenção ao que denominou capital intelectual (que dividiu em três categorias: incorporado, objetivado e institucionalizado) e à rede de relações, buscando estabelecer metodologia para analisá-los. Fez uma aplicação desta metodologia para avaliar o grupo sob sua liderança, GEP (Grupo de Pesquisa Ensino e Prática de Relações Públicas), vinculado à Pontifícia Universidade Católica do Rio Grande do Sul (PUC/RS).

É importante salientar que as análises feitas a partir do Diretório dos Grupos de Pesquisa do CNPq encontram no momento sérias restrições pelo formulário adotado para o registro de informações dos grupos, que é incompleto, com pouco espaço para o detalhamento das informações. O recurso, utilizado pela professora Cláudia Moura e que também adotamos, Currículo Lattes dos pesquisadores para obtenção de informações adicionais nem sempre é bem sucedido porque esta base de dados também tem limitações. Muitas vezes, na consulta das informações dos pesquisadores no Lattes, foi difícil associar sua produção às linhas de pesquisas às quais estão vinculados nos grupos porque, em geral, lá figuram apenas os títulos de seus trabalhos.

\section{Metodologia}

O estudo buscou identificar e descrever os grupos de pesquisa em comunicação organizacional existentes no Brasil, tomando como base de dados o Diretório dos Grupos de Pesquisa do CNPq. A etapa de seleção dos grupos foi feita pelo autor deste trabalho, assim como a tabulação e a análise dos dados. A descrição dos grupos de pesquisa e as informações sobre os pesquisadores foram obtidas pelo trabalho conjunto do autor deste trabalho e de pesquisadores que integram seu grupo de pesquisa.

1 É natural que haja uma discrepância na identificação e seleção dos grupos de pesquisa porque os critérios utilizados em ambos os casos (a pesquisa de Cláudia Moura e o nosso) não são coincidentes, mas essa diferença aponta para a dificuldade real que se encontra na própria delimitação dos campos de comunicação organizacional e relações públicas. Além disso, fizemos a busca no diretório por palavras-chave distintas das utilizadas pela autora em seu estudo anterior.
O Diretório dos Grupos de Pesquisa se constitui em projeto desenvolvido pelo CNPq há mais ou menos uma década e reúne, em uma base de dados, os grupos de investigação em atividade no país. Os grupos de pesquisa que constam desta base de dados vinculam-se, conforme define o portal deste diretório, "a universidades, instituições isoladas de ensino superior, institutos de pesquisa científica, institutos tecnológicos e laboratórios de pesquisa e desenvolvimento de empresas estatais ou ex-estatais" mas não levam em conta aqueles que, eventualmente, podem estar localizados em empresas privadas.

O diretório traz informações básicas sobre os grupos de pesquisa, incluindo seus recursos humanos (pesquisadores, estudantes e corpo técnico) com indicação explícita do(s) seu(s) líder(es), as linhas de pesquisa, sua produção científica ou tecnológica e seu vínculo com as instituições que os certificam, ano de criação, além da especialidade do conhecimento a que se filiam e informações gerais sobre os setores de aplicação envolvidos.

O estudo foi realizado em quatro etapas, a saber: (a) identificação e seleção dos grupos no Diretório do CNPq; (b) coleta dos dados gerais sobre o grupo a partir da consulta à sua página no diretório: (c) busca de informações sobre os líderes e integrantes na página do grupo existente no diretório e no currículo Lattes de cada um deles; (d) tabulação dos dados obtidos; e finalmente (e) análise dos dados. Adotamos um protocolo básico para o levantamento das informações sobre os grupos e os representantes/líderes, com o objetivo de padronizá-las e de permitir comparações que foram estabelecidas a posteriori na etapa de análise. No caso da produção foram considerados os períodos de 2008 a 2011.

A primeira etapa - busca dos grupos no diretório do $\mathrm{CNPq}$ - foi realizada no último trimestre de 2011, a partir de palavras-chave reconhecidamente indicativas do campo da comunicação organizacional. Como ele é bastante abrangente, tendo em vista o conceito que adotamos para a comunicação organizacional, valemonos de oito expressões. Elas incorporaram não apenas as denominações genéricas do campo (comunicação empresarial, comunicação organizacional e comunicação corporativa) mas também competências e recortes específicos (relações públicas, jornalismo empresarial, assessoria de imprensa, comunicação institucional e comunicação mercadológica).

A busca no diretório do CNPq gerou, num primeiro momento com a utilização dessas palavras-chave, 438 grupos (276 apenas valendo-se da expressão "relações públicas"), mas 


\section{A investigação em comunicação organizacional no Brasil: uma leitura abrangente dos grupos de pesquisa cadastrados no CNPq}

alguns critérios estabelecidos desde o início fizeram com que esse conjunto ficasse substancialmente reduzido ao final.

Podemos citar, entre esses critérios:

- Considerar apenas os grupos que tinham como área predominante a Comunicação;

- Não aceitar os grupos de pesquisa que tinham apenas um pesquisador;

- excluir os grupos que não estavam atualizados há mais de um ano;

- ignorar o grupo cuja produção fosse nula ou praticamente inexistente na área;

- excluir os grupos cujos líderes não participam das linhas de pesquisa identificadas explicitamente com a comunicação organizacional;

- eliminar os grupos que, embora permanecessem depois da aplicação dos critérios anteriores, não tinham efetivamente linhas de pesquisa e produção identificadas com o campo.

A aplicação destes critérios reduziu o total de grupos obtido inicialmente a apenas 29 , e foram esses que efetivamente constituíram o universo deste estudo.

Depois de identificados estes grupos, valendo-se do protocolo padrão recorremos ao espaço dos grupos no diretório do CNPq, que nos permitiu encontrar uma série de informações (nome do grupo, data de fundação, localização, vínculo institucional, relação dos pesquisadores, estudantes e técnicos, linhas de pesquisa com ementa/ objetivos e informações gerais sobre os seus trabalhos e produção). As demais informações, referentes aos pesquisadores em particular (formação, maior titulação, produção bibliográfica etc.), foram obtidas diretamente do currículo Lattes. Para tanto, foram visitados os currículos dos 181 pesquisadores cadastrados nos 29 grupos de pesquisa analisados.

Os dados obtidos foram tabulados a partir das categorias estabelecidas no protocolo-padrão e mereceram, a posteriori, análise específica, originando os resultados principais que estão apresentados mais diante neste estudo.

A pesquisa sobre os grupos de pesquisa em comunicação organizacional, tendo em vista as categorias previamente definidas, contemplou cinco hipóteses básicas, a saber:
1. Os grupos de pesquisa em comunicação organizacional estão vinculados institucionalmente, em sua maioria, a instituições públicas (universidades estaduais ou federais) e localizam-se nas regiões sul e sudeste do Brasil;

2. Os grupos de pesquisa em comunicação organizacional foram criados recentemente e a sua maioria está em atividade há menos de 10 anos. Eles têm em média até no máximo três linhas de pesquisa, integradas por três a cinco pesquisadores cada uma. A maioria dos grupos incorpora em sua equipe estudantes e técnicos. Poucos grupos têm espaço ou canal específico para a divulgação dos seus resultados de pesquisa (blogs, sites ou portais, publicações etc.);

3. Os grupos de pesquisa refletem o perfil do campo e são integrados basicamente por pesquisadores do gênero feminino, com formação básica (graduação e pós-graduação) em Comunicação, embora com participação expressiva também de investigadores de outros campos, em particular das chamadas Ciências Sociais Aplicadas. Essa situação se repete também entre os líderes. Os líderes têm titulação em média maior do que os demais integrantes dos grupos de pesquisa e são no mínimo doutores;

4. Os líderes respondem por porcentagem expressiva da produção bibliográfica dos grupos de pesquisa e, em média, produzem mais no campo. Muitos integrantes dos grupos de pesquisa têm pouca e algumas vezes nenhuma produção específica em comunicação organizacional;

5. Os pesquisadores dos grupos de pesquisa têm uma participação expressiva em atividades de orientações de trabalhos (trabalhos de conclusão de curso na graduação ou em programas de lato sensu) e apenas um número menor deles (geralmente os mais titulados e os líderes) incluem em seu currículo dissertações e teses orientadas (estas últimas ainda em pequeno número).

\section{Principais resultados}

É possível sintetizar os principais resultados da pesquisa realizada levando-se em conta as hipóteses formuladas. Assim temos:

1. Os 29 grupos de pesquisa em comunicação organizacional, resultado do levantamento realizado, situamse prioritariamente na região sul e sudeste do Brasil $(86,21 \%$ do total), com um número pouco expressivo nas demais regiões: $6,90 \%$ na região centro-oeste e 


\section{A investigação em comunicação organizacional no Brasil: uma leitura abrangente dos grupos de pesquisa cadastrados no $\mathrm{CNPq}$}

$6,90 \%$ na região sudeste. Obedecendo aos critérios fixados estabelecidos para a pesquisa, não há nenhum grupo na região norte do país. Os três Estados com maior número de grupos são, pela ordem, São Paulo e Paraná (com 7 grupos cada) e Rio Grande do Sul (6). Mesmo nas regiões com maior número de grupos de pesquisa, há Estados que não os contemplam, como Rio de Janeiro (região sudeste), Mato Grosso, Mato Grosso do Sul e Tocantins (região centro-oeste). A maioria dos grupos de pesquisa está vinculada institucionalmente a universidades públicas estaduais e federais $(65,51 \%)$, sendo que 6 deles $(20,68 \%)$ estão vinculados a uma mesma instituição (Universidade Estadual de Londrina). Outras três universidades abrigam mais de um grupo de pesquisa: Universidade de São Paulo (3), Universidade Metodista de São Paulo (2) e Universidade Federal de Santa Maria (2);

2. A maioria dos grupos de pesquisa $(48,27 \%)$ estava em 2011, ano da realização do levantamento, com 5 anos ou menos de atividade, e apenas um deles tinha 10 anos ou mais. Não existia nenhum grupo de pesquisa em comunicação organizacional no século passado, ou seja, antes do ano 2000. A maioria deles (82,75\%) tem até 2 linhas de pesquisa $(55,1 \%$ com uma linha e $27,58 \%$ com duas linhas). Há três grupos de pesquisa que têm cinco ou mais linhas de pesquisa. No total, os grupos de pesquisa incluem 181 pesquisadores e um total de quase 60 linhas de pesquisa. Isso significa que há, em média, três pesquisadores por linha de pesquisa. No total, as linhas incorporam 189 estudantes e apenas 8 técnicos, o que dá uma média de 9,45 estudantes por grupo e de 0,27 técnicos por grupo. Um número muito reduzido de grupos tem um espaço ou canal específico para divulgação dos seus trabalhos.

3. Dos 181 pesquisadores que integram os grupos de pesquisa em comunicação organizacional, as mulheres constituem a maioria $(65,75 \%)$. Esse fato se repete, embora com menor intensidade, entre os líderes do grupo. Dos 40 líderes dos grupos, 55\% são mulheres e $45 \%$ são homens. ${ }^{2}$ A maioria dos pesquisadores tem formação básica na graduação em Comunicação $(72,93 \%)$, vindo a seguir os formados em Administração (8,29\%), Letras/Linguística (4,42\%), Economia (2,76\%), Ciências Sociais (2,20\%), Direito, Psicologia e História (1,66\%). Embora a maioria dos

${ }^{2}$ O número de líderes excede o número de grupos de pesquisa porque há grupos com mais de um líder. pesquisadores seja oriunda da área de Ciências Humanas ou Sociais, é importante registrar a presença de representantes de áreas aparentemente menos próximas, como Medicina, Arquitetura, Medicina Veterinária, Química, Engenharia e Agronomia, o que comprova a inter e multidisciplinaridade neste campo. A mesma situação se repete entre os líderes de grupo, visto que $76,19 \%$ são formados em Comunicação, com apenas $23,81 \%$ em outras áreas, das quais a metade em Administração. Os líderes têm a Comunicação como área de referência na pósgraduação: $72,50 \%$ têm mestrado em Comunicação, $80,00 \%$ têm doutorado em Comunicação e 100\% dos que têm pós-doutorado o fizeram em Comunicação. No caso da titulação stricto sensu (mestrado e doutorado), apenas têm importância, além da Comunicação, as áreas de Administração (10\% dos mestrados e pouco mais de $15 \%$ dos doutorados), Sociologia (7,5\% dos mestrados e 2,5\% dos doutorados) e Engenharia da Produção ( $5 \%$ dos mestrados e $2,5 \%$ dos doutorados). A titulação dos líderes é, em geral, maior do que a da média dos pesquisadores dos grupos. Assim, 83,33\% dos líderes têm doutorado e 14,28\% têm pós-doutorado. Considerando os demais pesquisadores (excluídos os líderes), estas porcentagens são de $60,99 \%$ com doutorado e 7,80\% com pósdoutorado. Do total dos pesquisadores (excluindo os líderes), $8,51 \%$ só têm a graduação completa e $4,26 \%$ têm apenas especialização;

4. Os líderes geralmente apresentam uma produção bibliográfica (autoria de livros, capítulos em coletâneas ou artigos em periódicos acadêmicos e científicos) superior aos demais pesquisadores dos seus grupos e, em muitos casos, chegam a responder por mais de $50 \%$ do total da produção de todo o grupo. Vejamos: dos 990 itens registrados de produção bibliográfica pelos pesquisadores que integram os grupos, $46,97 \%$ são de autoria dos líderes. Esta importância pode ser percebida a partir de cada indicador de produção, ou seja, os líderes são responsáveis por $37,30 \%$ dos livros publicados, por $48,51 \%$ dos capítulos em coletâneas e por $47,83 \%$ dos artigos em periódicos. Isso significa, considerando-se o total de produção bibliográfica, que a média de produção dos líderes dos grupos é de 11,57 itens e dos demais pesquisadores, de 3,37 itens. Além da maior produção, os líderes geralmente produzem mais no campo do que a média dos demais pesquisadores. Sua produção está centrada em comunicação organizacional: $82,76 \%$ dos líderes têm mais de $50 \%$ de sua produção bibliográfica no campo, enquanto que os demais pesquisadores dos grupos têm uma 


\section{A investigação em comunicação organizacional no Brasil: uma leitura abrangente dos grupos de pesquisa cadastrados no $\mathrm{CNPq}$}

porcentagem menor e há casos de pesquisadores que não têm qualquer produção no campo. ${ }^{3}$

5. Os pesquisadores que integram os grupos de pesquisa dedicam um tempo importante para a orientação de trabalhos em nível de graduação ou pós-graduação. Assim, os 181 pesquisadores cadastrados nos grupos, no período analisado, orientaram 1940 trabalhos, dos quais $66,39 \%$ eram de graduação, $25,51 \%$ de especialização e $8,09 \%$ de pós-graduação strictu sensu: dissertações (81,53\% deste total) e teses (18,47\% desse total). Considerando-se apenas os 40 líderes, a orientação contemplou 653 trabalhos, sendo $64,01 \%$ de graduação, $22,20 \%$ de especialização, $11,03 \%$ de mestrado e $2,76 \%$ de doutorado. Isso significa que os líderes, em média, orientaram mais (média de 15,55 trabalhos cada) do que os demais pesquisadores (média de 9,13 trabalhos cada), com grande supremacia no que diz respeito à orientação de dissertações e teses. Assim, os líderes foram, em média, responsáveis por uma média de 2,14 dissertações orientadas cada, enquanto que os demais integrantes dos grupos de pesquisa responderam por 0,47 cada. Os líderes responderam pela orientação de $62,07 \%$ dos doutorados concluídos.

\section{Conclusões e sugestões}

Embora existam 438 grupos cadastrados no CNPq e passíveis de recuperação por palavras-chave identificadas com o campo da comunicação organizacional, apenas uma porcentagem reduzida deles $(6,62 \%)$ tem como área predominante a Comunicação, apresenta produção significativa, dispõe efetivamente de linhas de pesquisa e objetivos focados em objetos do campo, ou que mereçam ser considerados como ativos e/ou relevantes (atualizados há pelo menos um ano no Diretório de Grupos, mais de um pesquisador etc.).

\footnotetext{
3 Embora as páginas dos grupos no Diretório dos Grupos de Pesquisa do CNPq apontem para sua produção, em outros itens optamos por considerar apenas a autoria de livros integrais, de capítulos em livros ou de artigos em periódicos acadêmicos e científicos. Descartamos a produção em outras publicações (jornais, revistas de informações gerais, portais, sites, blogs) e os trabalhos apresentados em eventos. É forçoso reconhecer que um número significativo de pesquisadores concentra sua produção nestes outros espaços, mas, pela dificuldade de avaliá-los, optamos por restringir nossa análise a apenas esses três indicadores de produção.
}

Há um desequilíbrio importante na localização dos grupos porque a maioria deles, como vimos, está localizada no sul e sudeste, com pouca participação das outras regiões do país. Isso significa que é preciso estimular a criação de grupos em determinadas regiões de modo que eles possam contemplar, no futuro, a análise da realidade local, permitindo que, desta forma, possamos dispor de um cenário que vislumbre uma perspectiva efetivamente nacional. É importante também promover uma maior descentralização dos grupos por universidades, visto que muitos estão abrigados em número pequeno de instituições. Para se ter uma idéia, apenas quatro universidades (Universidade Estadual de Londrina, Universidade de São Paulo, Universidade Federal de Santa Maria e Universidade Metodista de São Paulo) respondem por $44,83 \%$ dos grupos considerados neste estudo.

Será importante também que se estimule a formação de grupos nas universidades privadas que, até este momento, abrigam um número pequeno deles, o que contribuiria decisivamente para a ampliação dos trabalhos de investigação no campo da comunicação organizacional.

É possível perceber nitidamente que os grupos de pesquisa em comunicação organizacional foram constituídos recentemente (a maioria está em atividade há menos de cinco anos) e que, portanto, a produção poderá crescer significativamente no futuro, quando as equipes estiverem mais maduras e mais articuladas.

Ainda que a maioria dos grupos mantenha um número adequado de linhas de pesquisa, existem distorções importantes em alguns grupos que, com um número pequeno de pesquisadores, mantêm um conjunto excessivo de linhas, o que contribui para a fragmentação da produção e para a ausência de um foco específico, sobretudo levando-se em conta a amplitude do campo.

Os grupos incorporam um número significativo de estudantes, além de pesquisadores, e esse aspecto é positivo na medida em que esta aproximação pode favorecer o aumento da massa crítica na área e propiciar, a curto e médio prazos, a formação de novos pesquisadores. Ressalta-se, no entanto, o número reduzidíssimo de técnicos ou auxiliares, o que certamente obriga os pesquisadores a desenvolverem atividades de suporte, de secretaria etc., com o acúmulo de funções que certamente penalizam sua dedicação ao trabalho de investigação.

Por uma série de motivos, a maioria dos grupos não dispõe de espaços ou canais para a divulgação de seus trabalhos (ou do trabalho individual de seus pesquisadores) 


\section{A investigação em comunicação organizacional no Brasil: uma leitura abrangente dos grupos de pesquisa cadastrados no $\mathrm{CNPq}$}

e, desta forma, a visibilidade dos grupos fica prejudicada. Além disso, a não divulgação desta produção acaba dificultando a articulação/interação entre os diversos grupos de pesquisa sobre comunicação organizacional existentes no Brasil, o que, se realizada, permitiria a troca de informações e experiências e a realização inclusive de trabaIhos em conjunto.

A maioria dos pesquisadores (e mais ainda dos líderes dos grupos) cursou pós-graduação (tem doutorado inclusive), particularmente na área de Comunicação, embora, como pudemos verificar pelos dados descritos anteriormente, o campo acolha profissionais e competências de áreas diversas, muitas vezes distantes, se considerada a formação dos pesquisadores da área de Ciências Humanas e Sociais (ou Ciências Sociais Aplicadas), onde se insere a Comunicação Social. É recomendável inclusive esta inter e multidisciplinaridade porque o campo, pela sua abrangência, se consolida pela convergência de competências e conhecimentos em várias áreas (Administração, Sociologia, Letras/ Linguística etc). Na verdade, o perfil dos pesquisadores dos grupos acaba legitimando a chamada "mestiçagem" do campo da comunicação organizacional, facilmente percebida inclusive na prática do mercado, com executivos e gestores de Comunicação que têm formação básica em diversas áreas.

\section{Referências}

Associação Brasileira de Pesquisadores de Comunicação Organizacional e Relações Públicas (Abrapcorp). Disponível em: <http://www.abrapcorp.org.br>. Acesso em: 09 de fevereiro de 2012.

BragA, José Luiz; LoPes, Maria Immacolata Vassalo; Martino, Luiz Cláudio. Pesquisa empírica em Comunicação. São Paulo: Paulus, 2010.

Conselho Nacional de Desenvolvimento Científico E Tecnológico (CNPq). Diretório dos Grupos de Pesquisa no Brasil. Disponível em: <http://dgp.cnpq. $\mathrm{br} /$ buscaoperacional/>. Acesso em: 10 de março de 2014.

Duarte, Jorge; Barros, Antonio. Métodos e técnicas der pesquisa em Comunicação. São Paulo: Atlas, 2005.
A presença expressiva de mulheres como pesquisadoras e mesmo líderes dos grupos de pesquisa repete o que encontramos tanto na área docente do campo da comunicação organizacional como sobretudo no mercado, especialmente pela importância dos cursos de Relações Públicas que respondem, num primeiro momento, na graduação, pela formação da maioria dos futuros profissionais em comunicação organizacional. Seria interessante avaliar se a supremacia das mulheres se reflete necessariamente na temática dos objetos de investigação. Há pesquisadores que acreditam que existe uma "ciência feminina", ou seja, que há temas e focos de interesse que estão mais próximos da mulher, em função de sua inserção na cultura e na sociedade.

A pesquisa evidenciou, de maneira contundente, a relação das linhas de pesquisa com o perfil de interesse dos líderes que têm efetivamente influência decisiva na composição e perfil dos grupos (em alguns deles a maioria dos pesquisadores acaba tendo vínculo com os líderes, são seus orientandos e ex-orientandos, ou mantém com eles vínculo de relacionamento no trabalho etc.). Além disso, como pudemos constatar, a produção dos líderes é significativamente maior do que a da média dos demais pesquisadores nos grupos. Em alguns grupos a produção bibliográfica (livros, capítulos em livros e artigos em periódicos acadêmicos ou científicos) dos grupos depende estreitamente da produção dos líderes ou quase se confunde com ela.

DenCKer, Ada de Freitas Manetti; ViÁ, Sarah Chucid da. Pesquisa empírica em ciências humanas (com ênfase em comunicação). São Paulo: Futura, 2001.

Denzin, Norman K.; Lincoln, Yvonna S. (Orgs). O planejamento da pesquisa qualitativa: teorias $e$ abordagens. Porto Alegre: Artmed Editora, 2006.

Fragoso, Suely; Recuero, Raquel; Amaral, Adriana. Métodos de pesquisa para internet. Porto Alegre: Sulina, 2011.

Galerani, Gilceana Soares Moreira. Avaliação em Comunicação Organizacional. Brasília: Embrapa, 2006.

Hérbet, Michelle Lessard; GoyetTe, Gabriel; Boutin, Gérald. Investigação qualitativa: fundamentos e práticas. $2^{\mathrm{a}}$ ed. Lisboa: Instituto Piaget, 2005. 


\section{A investigação em comunicação organizacional no Brasil: uma leitura abrangente dos grupos de pesquisa cadastrados no $\mathrm{CNPq}$}

Kunsch, Margaria Maria Khrohling. Planejamento de Relações Públicas na Comunicação Integrada. São Paulo: Summus Editorial, 2003.

Lefèvre, Fernando; LefĖvre, Ana Maria Cavalcanti. $O$ discurso do sujeito coletivo: um novo enfoque em pesquisa qualitativa (Desdobramentos). Caxias do Sul: EDUCS, 2003.

LoPES, Maria Immacolata Vassalo. Pesquisa em Comunicação. São Paulo: Edições Loyola, 1990.

Maingueneau, Dominique. Análise de textos de Comunicação. São Paulo: Cortez Editora, 2001.

Moura, Cláudia Peixoto. Grupos de pesquisa em Relações Públicas e Comunicação Organizacional: uma proposta metodológica para a análise das práticas acadêmicas. Disponivel em: http://confibercom.org/anais2011/pdf/74.pdf. Acesso em 07 de fevereiro de 2012.

PHILLIPS, Jack J.; MyHILL, Monica; McDonough, James B. O valor estratégico dos eventos: como e por que medir ROI. São Paulo: Aleph, 2008.

POUPART, Jean et al. A pesquisa qualitativa: enfoques epistemológicos e metodológicos. Petrópolis:Editora Vozes, 2008.

Powell, Guy R; Groves, Steven W.; Dimos, Jerry. Retorno sobre o investimento em mídias sociais. Rio de Janeiro: Elsevier, 2011.
REY, Fernando González. Pesquisa qualititativa e subjetividade: os processos de construção da informação. São Paulo: Pioneira Thompson Learning, 2005.

Rosenwald, Peter J. Accountable Marketing: otimizando resultados dos investimentos em Marketing. São Paulo: Pioneira Thompson Learning, 2005.

Rüdıger, Francisco. Ciência social crítica e pesquisa em comunicação. São Leopoldo: Editora Unisinos, 2002.

Santaella, Lucia. Comunicação \& Pesquisa. São Paulo: Hacker Editores, 2001.

Silverman, David. Interpretação de dados qualitativos. $3^{a}$ ed. Porto Alegre: Artmed Editora, 2009.

Sterne, Jim. Métricas em mídias sociais. São Paulo: Nobel, 2011.

Terra, Carolina Frazon. Mídias sociais...e agora? São Caetano do Sul: Difusão Editora; Rio de Janeiro: Editora Senac, 2011.

Weber, Maria Helena; Bentz, Ione; Hohlfeldt, Antonio. Tensões e objetos da pesquisa em Comunicação. Porto Alegre: Sulina, 2002.

YANAZE, Mitsuro. Retorno de investimentos em Comunicação: avaliação e mensuração. São Caetano do Sul: Difusão Editora, 2010. 